\title{
Zeitaufgelöste Messungen mit einem LSPR-Sensorsystem am Beispiel des Spurenstoffs Diclofenac
}

\author{
Kevin Thomschke ${ }^{1}$, Marisa Rio ${ }^{2}$, Nadja Steinke ${ }^{1}$, Michael Seidel ${ }^{3}$, Fabian Dortu', Damien Bernier ${ }^{4}$, Steffen \\ Howitz ${ }^{5}$, Thomas Härtling ${ }^{1,6}$ und Christiane Schuster ${ }^{1}$

\begin{abstract}
${ }^{1}$ Fraunhofer Institut für keramische Technologien und Systeme IKTS, Dresden, Deutschland 3Institut für Wasserchemie und Chemische Balneologie, TU München, München, Deutschland ${ }^{4}$ MULTITEL asbl., Mons, Belgien

${ }^{5}$ Gesellschaft für Silizium-Mikrosysteme $\mathrm{mbH}$, Radeberg, Deutschland

${ }^{6}$ Institut für Festkörperelektronik, TU Dresden, Dresden, Deutschland

Kontakt: kevin.thomschke@ikts.fraunhofer.de
\end{abstract} \\ ${ }^{2}$ Fraunhofer Center for Smart Agriculture and Water Management - AWAM, Vila Real, Portugal
}

\section{Einleitung}

Seit Jahren gelangen Rückstände umweltschädlicher Pharmazeutika über Abwässer in die Umwelt, da viele Substanzen nicht oder nur unzureichend in konventionellen Kläranlagen entfernt werden können [1]. Mangels geeigneter Messsysteme ist insbesondere die Vor-Ort-Überwachung der Schadstoffe, die im $\mathrm{ng} / \mathrm{L}-\mathrm{bis} \mu \mathrm{g} / \mathrm{L}-$ Bereich vorliegen, derzeit nicht möglich.

Gegenwärtige Methoden zum analytischen Spurenstoffnachweis, z.B. mittels Hochleistungsflüssigkeitschromatografie, sind kostspielig und erfordern eine Laborumgebung [2]. Damit ist insbesondere keine instantane Überwachung von Schwellenwerten im Sinne einer Wächterfunktion möglich, um bei Überschreitung weitere Verfahrensstufen in der Kläranlage oder zusätzliche quantitative Analysen einzuleiten. Aus diesem Grund wird hier ein Biosensor vorgestellt, mit welchem die Wasserqualität anhand des Schmerzmittels Diclofenac (DCF) als Leitsubstanz für eine Schwellenwertbestimmung direkt beurteilt werden kann.

Für die Detektion von DCF in wässrigen Medien nutzt das vorgestellte Sensorsystem ein nanostrukturiertes Goldsubstrat, auf dessen Oberfläche lokalisierte Oberflächenplasmonen (localized surface plasmon, LSP) angeregt werden. Im Resonanzfall (localized surface plasmon resonance, LSPR) sind diese LSP sensitiv gegenüber Änderungen des Brechungsindexes in unmittelbarer Nähe der Oberfläche $(<50 \mathrm{~nm})$, welche als spektrale Verschiebung des Transmissionsspektrums ausgelesen werden können.

Die Nanostruktur fungiert dabei als Transduceroberfläche, über welche mittels einer Oberflächenfunktionalisierung Molekülanbindungen anhand der dabei auftretende oberflächennahen Brechzahländerung zeitaufgelöst detektiert werden. An der Oberfläche der Nanostruktur werden DCF-Moleküle immobilisiert. Durch einen Anti-DCF Antikörper [3], welcher an die immobilisierten DCF-Moleküle bindet, wird die Brechzahländerung induziert.
Das Sensorsubstrat wird im Transmissionsaufbau optisch durch LEDs angeregt. Mittels einer spektralen Ausleseeinheit (Multitel asbl., Mons, Belgien) werden optische Spektren ausgelesen. Die Besonderheit dabei ist, dass diese kontinuierlich in Zeitabständen von ca. $1 \mathrm{~s}$ automatisiert aufgenommen werden. Ein integrierter Algorithmus wertet die optischen Spektren in Echtzeit bzgl. ihrer Resonanzlage aus. Die Messergebnisse werden in einem Sensorgramm als Lage der plasmonischen Resonanz über der Zeit dargestellt. Somit kann der zeitliche Verlauf der Molekülanbindung auf der Oberfläche aufgezeigt werden. Die Einbindung des Sensorsubstrats in ein mikrofluidisches System (MicCell, GeSiM mbH Radeberg, Deutschland) gewährleistet zudem die Automatisierung und Reproduzierbarkeit der Messung.

Die Verwendung des LSPR-Sensorsystems zur Detektion von konkreten DCF-Konzentrationen wurde bereits nachgewiesen [4], dabei ergab sich ein Arbeitsbereich von 4,7-12,4 $\mu \mathrm{g} / \mathrm{L}$ [5]. Diese Art der Messung mittels indirektem Assay beruht auf einer Vorinkubation der Probe für einen gewissen Zeitraum, so dass die Messung nicht unmittelbar geschieht.

In diesem Beitrag soll die Anwendung des Sensorsystems zur Schwellwertüberwachung gezeigt werden. Dazu wird der zeitliche Verlauf des DCF-Gehaltes eines Analyten kontinuierlich überwacht. Zur Anwendung kommt hierfür ein Verdrängungsassay, in welchem die Oberfläche des nanostrukturierten Goldsubstrats mit einem Anti-DCF Antikörper abgesättigt wird, wodurch eine maximale Verschiebung der Lage der plasmonischen Resonanz erreicht wird. Im nächsten Schritt wird der Analyt über die Sensoroberfläche geführt. Es wird erwartet, dass sich abhängig von der DCF-Konzentration in der Probe Antikörper von der Oberfläche ablösen und an das DCF in der Flüssigkeit binden. Dies ist unmittelbar über eine Signaländerung im Sensorgramm nachweisbar. Über die Dynamik der Signaländerung soll so mit dem Sensorsystem detektiert werden, ob Maßnahmen im Wasseraufbereitungsprozess notwendig sind, z.B. 
Medienseparation, Zuschalten weiterer Reinigungsstufen, Filteraustausch oder weitere Analytik.

\section{Methoden und Materialien}

\section{Aufbau des LSPR-Sensorsystems}

Die Herstellung der nanostrukturierten Goldsubstrate mittels Nanoimprint-Lithografie und anschließender Gold-Besputterung, sowie die Oberflächenfunktionalisierung erfolgt wie in [4] dargelegt.

Das Sensorsubstrat umfasst zwei mikrofluidische Kanäle mit je zwei nanostrukturierten Bereichen. Eine dieser nanostrukturierten Flächen wird DCF-oberflächenfunktionalisiert und dient als Messfläche. Um äußere Einwirkungen wie Temperatureffekte oder unspezifische Bindungen auszugleichen, wird das Signal der Messfläche mit dem Signal einer zweiten passivierten nanostrukturierten Fläche in der Signalauswertung referenziert.

Das Sensorsubstrat wird in eine Trägerplattform eingesetzt und über eine Anschlussplatte mit dem mikrofluidischen System verbunden. Die Anregung der LSPs erfolgt über LEDs mit einer zentralen Wellenlänge von $875 \mathrm{~nm}$. Unterhalb der Trägerplattform wird das transmittierte Licht jedes nanostrukturierten Bereiches mit je einer optischen Faser aufgefangen und zur spektralen Analyse in die Ausleseeinheit (BIOPHOTON020, Multitel asbl.) geleitet. Daraus werden durch ein Transmissionsgitter vier parallele Dispersionsspektren erzeugt, welche mit Hilfe eines CMOSSensors in digitale Pixelreihen umgewandelt werden. Die so aufgenommenen Bilder werden von dem integriertem Linux-Betriebssystem verarbeitet und zunächst auf einen spektralen Bereich zwischen $750-950 \mathrm{~nm}$ begrenzt. Je Faser wird eine Pixelreihe mit 3088 Pixeln aufgenommen, aus welchen anschließend anhand der Intensität der Pixel die optischen Spektren extrahiert werden. Die Bestimmung der Resonanzlage erfolgt über die Anpassung einer Lorentz-Funktion mittels Levenberg-Marquardt-Algorithmus. Der Scheitelpunkt der angepassten LorentzFunktion entspricht der Resonanzwellenlänge zum Zeitpunkt des aufgenommenen Spektrums und wird als Datenpunkt im Sensorgramm angezeigt.

\section{Ablauf einer Messung}

Zu Beginn der Messsequenz wird zunächst die plasmonische Resonanz in phosphatgepufferter Salzlösung (PBS, pH 7,4) aufgenommen. Diese dient als Basislinie und ist der Ausgangswert für den weiteren Verlauf der Messung. Dafür werden $2 \mathrm{ml}$ PBS mit einem konstanten Volumenstrom von $10 \mu \mathrm{l} / \mathrm{s}$ über das Substrat gespült. Zur Absättigung der Oberfläche wird ein monoklonaler Anti-DCF Antikörper mit einer Antikörperkonzentration von $4 \mu \mathrm{g} / \mathrm{l}$ in $1000 \mu \mathrm{l}$ PBS über die Oberfläche geführt. Daraufhin werden $1000 \mu \mathrm{l}$ Probenvolumen, die den Analyten DCF in Konzentrationen von 50,100, 250 und $500 \mu \mathrm{g} / \mathrm{L}$ in
PBS enthalten, mit einem Volumenstrom von 1,5 $\mu \mathrm{l} / \mathrm{s}$ durch den mikrofluidischen Kanal geleitet. AbschlieBend wird nochmals PBS durch den Kanal gespült, um die Resonanzlage nach der DCF-haltigen Probe aufzuzeichnen, welche als verschobene Basislinie den Endwert der Messung darstellt.

\section{Ergebnisse}

\section{Auswertung der Sensorgramme}

Die zu Beginn jeder Messung aufgenommene Basislinie dient als Ausgangswert zur Bestimmung der Verschiebung der plasmonischen Resonanz. Im Folgenden steigt der Kurvenverlauf im Sensorgramm durch die Antikörperanbindung zunächst stark an bis alle Bindungsstellen nahezu abgesättigt sind (hier verkürzt, um die eingesetzte Antikörpermenge zu reduzieren). Die Experimente zeigen, dass unter den gegeben Assay-Bedingungen mit dem monoklonalen Antikörper eine maximale Resonanzverschiebung von ca. 2,1 nm erreicht wird.

Durch das anschließende Durchströmen des mikrofluidischen Kanals mit einer DCF-haltigen Probe kommt es zum Abfallen des Messsignals. Das immobilisierte DCF an der Oberfläche steht mit dem DCF in der freien Phase in Konkurrenz um die Bindungsstelle des Antikörpers. Dabei ist das DCF in der freien Phase der bevorzugte Bindungspartner, wodurch sich Antikörper von der Oberfläche ablöst, an das DCF in der Probe bindet und von der strömenden Probe abtransportiert wird. Die Ablösung, oder Verdrängung, ist proportional zur DCF-Konzentration in der Probe [6]. Dies wird durch Messergebnisse bestätigt. Die Signaländerung ist konzentrationsabhängig. Die Sensorgramme für die DCF-Konzentrationen $50,100,250,500 \mu \mathrm{g} / \mathrm{l}$ sind in Abb. 1 zu sehen.

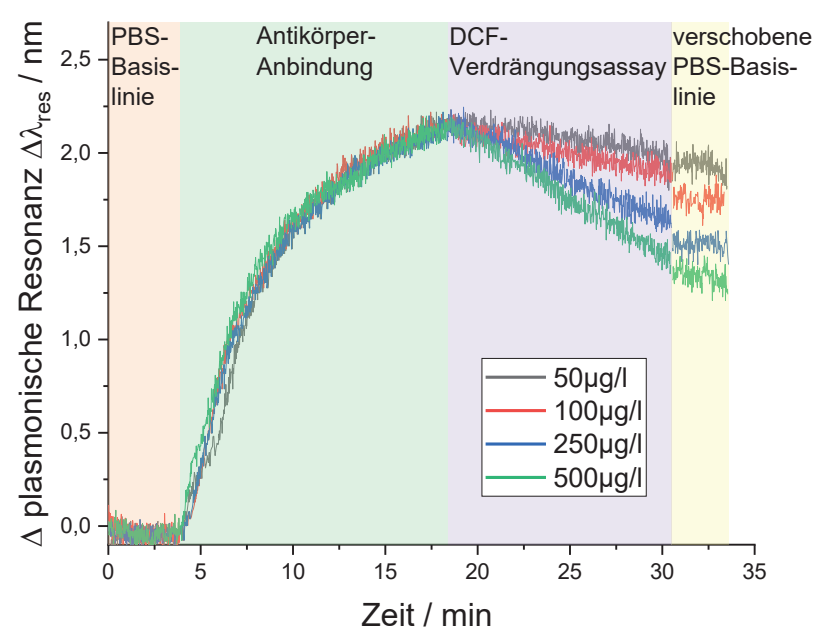

Abb. 1: referenziertes Sensorgramm für zeitaufgelöste Antikörper-Anbindung und -Verdrängung.

Der Signalabfall kann im DCF-Verdrängungsassay als Maß für die Dynamik der Signaländerung 
herangezogen werden. Dieser wird hier linear angepasst. Weiterhin kann die Signaländerung als Differenz zwischen der Resonanzlage nach der Antikörperanbindung (maximale Resonanzverschiebung) und der Resonanzlage nach dem DCF-Verdrängungsassay (verschobene PBS-Basislinie) beschrieben werden. Die Ergebnisse werden in Tab. 1 zusammengefasst.

Tab. 1: DCF-Konzentrationen $c_{D C F}$ und deren Signaländerungen (lineare Steigung $p$ und Differenz der Reso-

\begin{tabular}{|c|c|c|}
\hline $\begin{array}{c}c_{D C F} \\
{\left[{ }^{\mu g} /{ }_{l}\right]}\end{array}$ & {$\left[\begin{array}{c}p \\
{[n m / \min ]}\end{array}\right.$} & $\begin{array}{c}\left(\lambda_{\text {res,max }}-\lambda_{\text {res }, D C F}\right) \\
{[\mathrm{nm}]}\end{array}$ \\
\hline 50 & -0.015 & 0.168 \\
\hline 100 & -0.02 & 0.344 \\
\hline 250 & -0.047 & 0.589 \\
\hline 500 & -0.062 & 0.760 \\
\hline
\end{tabular}

Die Dynamik der Signaländerung liegt bei einer DCFKonzentration von $50 \mu \mathrm{g} / \mathrm{l}$ bei $-0.015 \mathrm{~nm} / \mathrm{min}$, während das Signal sich um $0.168 \mathrm{~nm}$ verringert. Dies entspricht ca. $8 \%$ der hier maximalen durch den Antikörper initiierten Resonanzverschiebung. Bei höheren Konzentrationen wird der Unterschied deutlicher. Bei einer DCF-Konzentration von $500 \mu \mathrm{g} / \mathrm{l}$ erhöht sich der Signalabfall auf $-0.062 \mathrm{~nm} / \mathrm{min}$. Entsprechend erhöht sich der Signalunterschied auf $0.76 \mathrm{~nm}$. Dies entspricht einem Signalabfall von ca. $36.2 \%$.

\section{Diskussion}

Die Experimente haben gezeigt, dass sich das LSPR-Sensorsystem zur Schwellwertüberwachung eignen kann. Die Signaländerung ist wie erwartet konzentrationsabhängig. Allerdings liegen die in diesem Beitrag nachgewiesene Konzentrationsstufen über dem für den Anwendung in einer Kläranlage sinnvollen Bereich. Abwässer von Kläranlagen weisen gewöhnlich einen DCF-Gehalt von 1 bis $10 \mu \mathrm{g} / \mathrm{l}$ auf [7].

Es wird vermutet, dass durch die hohe Bindungsaffinität des verwendeten Antikörpers die Bindung des an der Oberfläche immobilisierten Antigen-Antikörper-Komplexes zu stark ist, um genügend Antikörper abzulösen und so auch bei geringeren Konzentrationen eine entsprechende Signalverminderung zu bewirken. Die Bindungsaffinität wird durch die Dissoziationskonstante beschrieben. Diese liegt bei dem vorliegenden monoklonalen Antikörper bei $1.5^{\star} 10^{-10} \mathrm{M}$ [3]. Ein Antikörper mit geringerer Bindungsaffinität könnte das Verdrängungsassay auch für geringere Konzentrationen einsetzbar machen. Dies soll Gegenstand zukünftiger Experimente sein.

Die Weiterverfolgung dieser Art der Messung ist jedoch vielversprechend, denn sie birgt einen Vorteil gegenüber der direkten Messung der DCF-
Konzentration mittels indirektem Assay. Die Probe muss keiner Vorbehandlung unterzogen werden und kann unmittelbar vermessen werden. Idealerweise wird die Probe bzw. das Abwasser kontinuierlich vermessen. So können bei auftretenden Konzentrationsspitzen unmittelbar Gegenmaßnahmen getroffen werden.

Der Anwendungsbereich ist dabei nicht auf DCF beschränkt, denn mit anderen Oberflächenfunktionalisierungen kann das Sensorsystem zukünftig ebenfalls zur Detektion von anderen Molekülen, auch in Form von Multiplexing, zum Einsatz kommen.

\section{Literatur}

[1] SATHISHKUMAR, P., MEENA, R. A. A., PALANISAMI, T., ASHOKKUMAR, V., PALVANNAN, T., \& GU, F. L.: Occurrence, interactive effects and ecological risk of diclofenac in environmental compartments and biota - a review. In: Science of the Total Environment, 698 (2020), 134057

[2] HERNANDO, M. D., HEATH, E., PETROVIC, M., AND BARCELÓ, D.: Trace-level determination of pharmaceutical residues by LC-MS/MS in natural and treated waters. A pilot-survey study. In: Analytical and Bioanalytical Chemistry, 385(2006), Nr. 6, S. 985-991

[3] HÜBNER, M.: Development of Immunological Methods for the Detection of Micropollutants in Fresh Water Samples. Technische Universität München, Dissertation, 2015

[4] STEINKE, N.; DÖRING, S.; WUCHRER, R.; KROH, C.; GERLACH, G. \& HÄRTLING, T.: Plasmonic sensor for on-site detection of diclofenac molecules. In: Sensors and Actuators B: Chemical, 288 (2019), S. 594-600

[5] THOMSCHKE, K.: Validation of a plasmonic immunosensor for the on-site detection of diclofenac. Hochschule für Wirtschaft und Technik Dresden, Masterarbeit, 2021

[6] GELINSKY-WERSING, D.: Kinetisches Modell für die Prozessanalyse von Displacement-Assays mit monound bivalenten Antikörpern. Technische Universität Dresden, Dissertation, 2018

[7] REEMTSMA, T., WEISS, S., MUELLER, J., PETROVIC, M., GONZÁLEZ, S., BARCELO, D., VENTURA, F., \& KNEPPER, T. P.: Polar pollutants entry into the water cycle by municipal wastewater: A European perspective. In: Environmental Science \& Technology, 40 (2006), Nr. 17, S. 5451-5458 